\title{
Análisis de los defectos congénitos en Colombia, 2015-2017
}

\section{Analysis of congenital defects in Colombia from 2015 to 2017}

\author{
Greace-Alejandra Avila-Mellizoํㅜ ${ }^{1}$ Nathaly Rozo-Gutierrez ${ }^{1}$; Diana-Alexa Forero-Motta ${ }^{1}$
}

Forma de citar: Avila-Mellizo GA, Rozo-Gutierrez N, Forero-Motta DA. Análisis de los defectos congénitos en Colombia 20152017. Rev Univ Ind Santander Salud. 2019; 51(3): 200-206. doi: http://dx.doi.org/10.18273/revsal.v51n3-2019003 (c) (1)

\section{Resumen}

Introducción: Los defectos congénitos son alteraciones del desarrollo que se generan en la vida intrauterina y tienen un alto impacto en la mortalidad infantil. La Organización Panamericana de Salud ha definido que la vigilancia en salud pública es una de las herramientas fundamentales para obtener estimaciones más precisas sobre la prevalencia del evento. Metodología: Estudio descriptivo retrospectivo con base en la notificación individual semanal de casos al Sistema de Vigilancia en Salud Pública (Sivigila) del evento 215 - Defectos congénitos de los años 2015, 2016 y 2017. Resultados: La notificación de defectos congénitos en Colombia ha aumentado un 39,9\% entre 2015 y 2017 con una tasa de prevalencia para el último año de 106,6 casos por 10.000 nacidos vivos. Las tasas de prevalencia más altas se encontraron en cuatro grupos diferentes: 1) menores de sexo masculino, 2) pertenencia étnica indígena, 3) hijos de madres no aseguradas al sistema de salud, 4) hijos de madres con edades mayores de 35 años. Así mismo, los defectos congénitos del sistema osteomuscular, sistema circulatorio y sistema nervioso central presentaron la mayor tasa de prevalencia. Discusión: Las mayores tasas de prevalencia identificadas en algunos grupos poblacionales son consistentes con otros estudios realizados en la región, y a su vez pueden ser relacionados con las condiciones de vulnerabilidad que limitan posiblemente la falta de oportunidad y acceso a los servicios de salud.

Palabras clave: Anomalías congénitas; Vigilancia en salud pública; Mortalidad infantil; Discapacidades del desarrollo; Colombia; Embarazo.

\begin{abstract}
Introduction: Congenital defects, which are generated in intrauterine life, are alterations of the development and have a high impact on infant mortality. PAHO states that public health surveillance is one of the fundamental tools to obtain more accurate estimates of the prevalence of the event. Methodology: A retrospective, descriptive study based on the weekly individual notification of cases to the Public Health Surveillance System (SIVIGILA) of the 215 event - congenital defects identified during the years 2015, 2016 and 2017. Results: The notification of congenital defects in Colombia increased by 39.9\% between 2015 and 2017 with a prevalence rate of 106.6 cases per 10,000 living births during 2017. The highest prevalence rates of congenital defects were found in four different groups:
\end{abstract}

1. Instituto Nacional de Salud. Bogotá D.C. Colombia

Correspondencia: Greace Alejandra Avila Mellizo. Dirección: Calle 26 No. 51-20 Bogotá. Teléfono: (+571) 2207700 Ext. 1476. Correo electrónico: gavilam@ins.gov.co 
1) under age males, 2) people with indigenous ethnicity, 3) children whose mothers are not insured to the health system, 4) children whose mothers are older than 35 years old. Likewise, congenital defects of the musculoskeletal system, the circulatory system and the central nervous system evidenced the highest prevalence rate. Discussion: The highest prevalence rates identified in some population groups are consistent with other studies conducted in the region, and they can also be related to conditions of vulnerability that possibly limit the opportunities to access the different health services.

Keywords: Congenital abnormalities; Public health surveillance; Infant mortality; Developmental disabilities; Colombia; Pregnancy.

\section{Introducción}

Los Defectos Congénitos (DC) son definidos como alteraciones del desarrollo (morfológicos, estructurales, funcionales $\mathrm{o}$ moleculares) presentes al nacer, aunque sus manifestaciones pueden ser detectadas posteriormente ${ }^{1}$. Los Defectos Congénitos Mayores (DCM), tienen un compromiso funcional importante para la vida del individuo, con afectación clínica, corporal y a nivel social por la discapacidad generada que requieren de atención temprana e integral ${ }^{2,3}$.

La Organización Panamericana de la Salud desde el 2016 invitó a los países miembros a fortalecer la vigilancia de los defectos de nacimiento como herramienta para la obtención de estimaciones más precisas de la prevalencia de la microcefalia y otras anomalías congénitas en recién nacidos. El sistema de vigilancia de anomalías congénitas de Europa (EUROCAT, por sus siglas en inglés) reporta una prevalencia de 215,4 por 10.000 nacidos vivos ${ }^{4}$, por lo que los ubica dentro de las primeras causas de muerte en los niños menores de cinco años.

El Estudio Colaborativo Latinoamericano de Malformaciones Congénitas (ECLAMC) reporta una tasa de prevalencia de defectos congénitos en la región de $2,6 \%$, siendo Brasil $(4,0 \%)$ y Chile $(3,1 \%)$ los países con las tasas más altas. En Chile los principales defectos congénitos son la cardiopatía congénita $(35,3$ casos por 10.000 nacidos vivos) y polidactilia $(23,4$ casos por 10.000 nacidos vivos) ${ }^{6}$.

Este problema de salud pública, requiere de atención multidisciplinaria desde la etapa preconcepcional con la asesoría genética y estudio de factores de riesgo; en la etapa prenatal con los tamizajes y seguimientos ecográficos para la detección temprana que posibilite la planeación del parto de acuerdo con el defecto congénito identificado, así como garantizar la atención temprana e idónea, el tratamiento y el seguimiento integral para la discapacidad ${ }^{7}$. Es importante tener en cuenta que su diagnóstico temprano posibilita contribuir en la reducción de la mortalidad, ya que ocasiona cerca del 20 a $30 \%$ de las muertes infantiles y 30 a $50 \%$ de las muertes postneonatales. De la proporción que logra sobrevivir, cerca del $25 \%$ desarrollará una discapacidad física o mental y el restante $50 \%$ tendrá una perspectiva favorable ${ }^{8}$.

El objetivo del estudio fue describir el comportamiento de los defectos congénitos en Colombia entre 2015 y 2017, teniendo en cuenta sus características sociales, demográficas y clínicas.

\section{Metodología}

Diseño: estudio descriptivo retrospectivo. Los datos fueron obtenidos de fuente primaria, por medio de la notificación individual semanal de casos al sistema de vigilancia en salud pública (Sivigila) del evento 215 Defectos congénitos de los años 2015, 2016 y 2017, realizada por las unidades primarias generadoras de datos (UPGD) a nivel nacional, las cuales identificaron y configuraron el caso para malformación congénita.

Los datos se sometieron a un proceso de depuración, verificando el tener datos completos, consistencia y veracidad. Se realizó la validación y se eliminaron los casos con ajuste 6 y D, es decir los casos descartados por error de notificación o porque no cumplían los criterios para defectos congénitos. Para los casos clasificados como confirmados por laboratorio o clínica se realizó la verificación de la codificación CIE -10 de acuerdo con la descripción del defecto registrada.

El análisis se realizó mediante estadística descriptiva. Se calcularon frecuencias absolutas y medidas de tendencia central. Se calculó la tasa de prevalencia, expresada como el número de casos de defectos congénitos notificados, sobre la población expuesta al riesgo (nacidos vivos) ponderada por la constante 10.000 (tomada de los parámetros internacionales). Los denominadores para el cálculo de la prevalencia 
corresponden los nacidos vivos de 2018 reportados por el Departamento Administrativo Nacional de Estadística (DANE).

\section{Resultados}

La notificación de defectos congénitos en Colombia ha aumentado un $39,9 \%$ entre 2015 y 2017 , pasando de 4.994 a 6.985 casos. Así mismo, la tasa de prevalencia por 10.000 nacimientos pasó de 75,6 a 106,6. Por área de residencia, se encontró una notificación de casos más alta en los hijos de madres residentes en las áreas urbanas, cuatro veces mayor en promedio, comparado con los hijos de madres residentes en áreas rurales, siendo también más alta la tasa de prevalencia en el área urbana en el último año en estudio (Tabla 1).

Con relación a las variables demográficas y sociales se encontró una mayor notificación de casos y tasa de prevalencia en el sexo masculino. Con respecto al régimen de afiliación a salud las tasas más altas se reportan en la población no asegurada seguida de la población del régimen subsidiado. Por pertenencia étnica la mayor tasa de prevalencia, en 2015 y 2017, se reportó en población indígena. Finalmente, por grupo de edad de la madre se observó que la mayor proporción de defectos congénitos se notificó en hijos de madres entre los 20 y 29 años, pero la tasa de prevalencia más alta se encontró en mujeres de 35 y más años, con tasas de hasta 267,5 casos por cada 10.000 nacidos en mujeres de 40 y más años (Tabla 2).

Tabla 1. Comportamiento de la notificación y tasa de prevalencia de defectos congénitos mayores según área de residencia de la madre, Colombia, 2015 - 2017.

\begin{tabular}{ccccccc}
\hline \multirow{2}{*}{ Año } & \multicolumn{2}{c}{ Total } & \multicolumn{2}{c}{ Urbano } & \multicolumn{2}{c}{ Rural } \\
\cline { 2 - 7 } & Casos & Tasa & Casos & Tasa & Casos & Tasa \\
\hline 2015 & 4.994 & 75,6 & 3.934 & 74,7 & 1.060 & 79,0 \\
2016 & 6.561 & 101,4 & 5.171 & 100,4 & 1.390 & 105,2 \\
2017 & 6.985 & 106,6 & 5.633 & 109,2 & 1.352 & 96,8 \\
\hline
\end{tabular}

Fuente: Sivigila, INS. 2015-2017. Estadisticas vitales, DANE. 2015 $-2017$.

Tabla 2. Comportamiento de la notificación y tasa de prevalencia de defectos congénitos mayores según variables demográficas y sociales, Colombia, $2015-2017$.

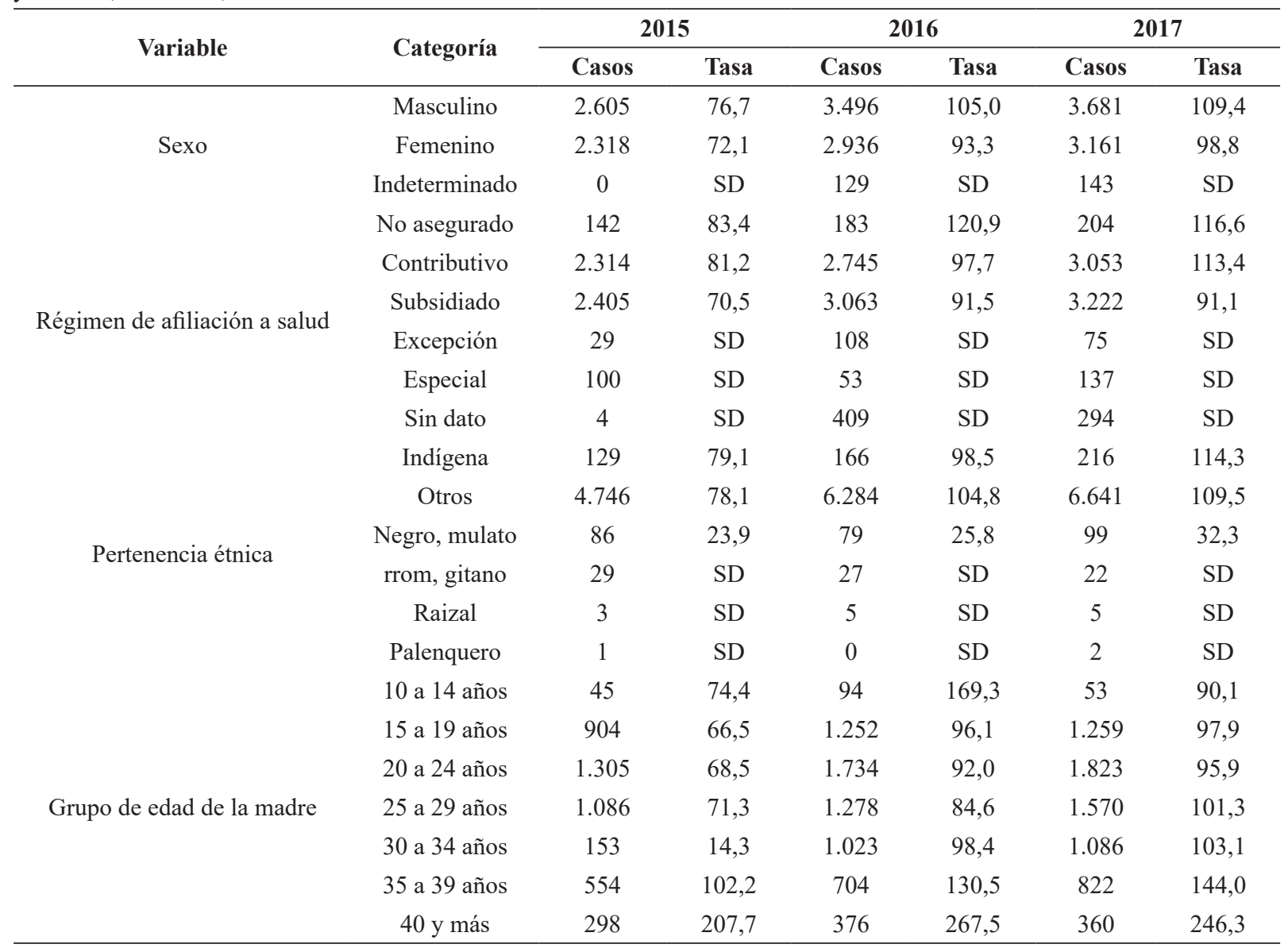

Fuente: Sivigila, INS. 2015-2017. Estadisticas vitales, DANE. 2015 - 2017 
Por entidad territorial de residencia para 2017 la mayor tasa de prevalencia por 10.000 nacidos vivos se encontró en Vaupés $(286,7)$, Vichada $(226,4)$, Casanare $(206,5)$, Guainía $(180,1)$ y Quindío $(174,3)$. Mientras que las menores tasas se encontraron en los departamentos de Magdalena y Chocó (para los tres años en estudio), y en La Guajira, Atlántico y San Andres (para dos de los tres años en estudio) con tasas inferiores a 60 casos por 10.000 nacidos (Gráfica 1).

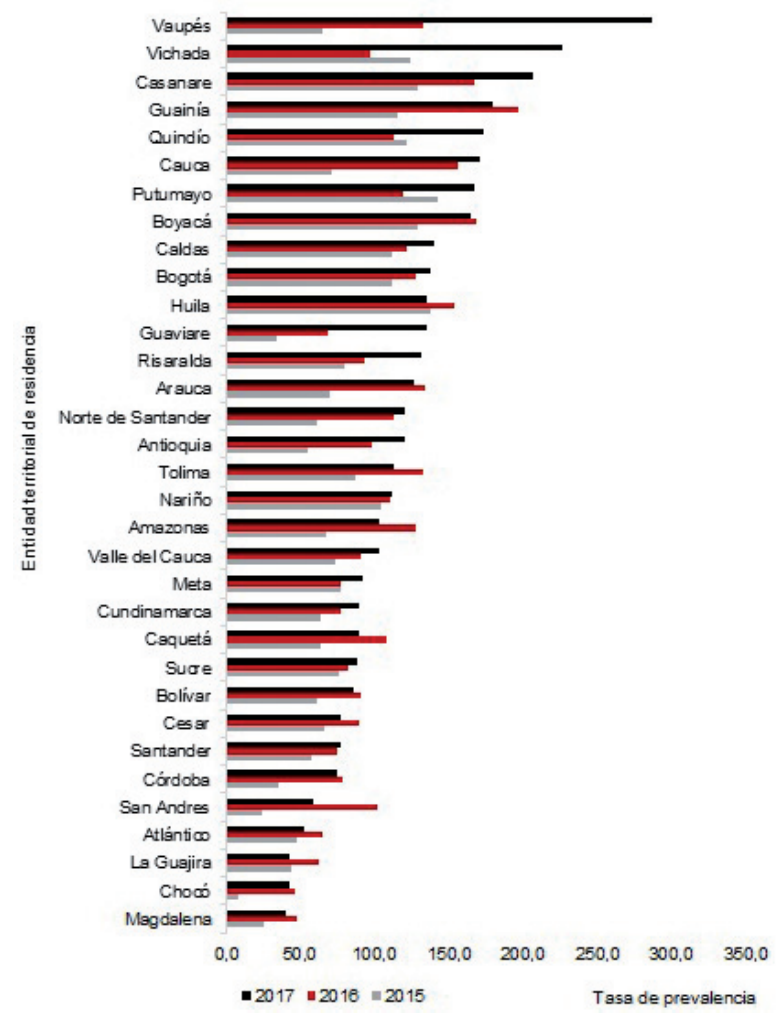

Gráfica 1. Comportamiento de la notificación y tasa de prevalencia de defectos congénitos mayores por entidad territorial de residencia de la madre, Colombia, 2015 - 2017. Fuente: Sivigila, INS. 2015-2017. Estadisticas vitales, DANE. $2015-2017$

Los defectos congénitos fueron agrupados de acuerdo con la clasificación CIE 10, encontrando la mayor tasa de prevalencia en los defectos del sistema osteomuscular, seguido de los defectos del sistema circulatorio y los defectos congénitos del sistema nervioso (Tabla 3).

Los defectos que se encontraron con mayor frecuencia en el grupo de sistema osteomuscular fueron la polidactilia (Q69) y la deformidad congénita de pies (Q66), en el grupo de defectos del sistema circulatorio fueron las malformaciones congénitas de los tabiques cardiacos (Q21) y malformaciones congénitas de las grandes arterias (Q25), y en el grupo de defectos congénitos del sistema nervioso la mayor frecuencia se encontró en microcefalia (Q02) e hidrocéfalo congénito (Q03).

De acuerdo con la condición final el 83,2\% de los casos fueron notificados con condición final vivo. La tasa de mortalidad ha presentado una disminución en los tres años en estudio, pasando de $17,4 \%$ en 2015 a $15,0 \%$ en 2017 (Tabla 4).

Tabla 3. Comportamiento de la notificación y tasa de prevalencia de defectos congénitos mayores por grupo CIE 10, Colombia, 2015- 2017.

\begin{tabular}{|c|c|c|c|c|c|c|}
\hline \multirow{2}{*}{$\begin{array}{l}\text { Grupos CIE } 10 \text { de } \\
\text { malformaciones } \\
\text { congénitas }\end{array}$} & \multicolumn{2}{|c|}{2015} & \multicolumn{2}{|c|}{2016} & \multicolumn{2}{|c|}{2017} \\
\hline & Casos & Tasa & Casos & Tasa & Casos & Tasa \\
\hline $\begin{array}{l}\text { Sistema nervioso } \\
\text { (Q00-Q09) }\end{array}$ & 640 & 9,7 & 1306 & 20,2 & 1063 & 16,2 \\
\hline $\begin{array}{l}\text { Ojo, oído, cara y } \\
\text { cuello (Q10-Q18) }\end{array}$ & 216 & 3,3 & 275 & 4,3 & 273 & 4,2 \\
\hline $\begin{array}{l}\text { Sistema circulatorio } \\
\text { (Q20-Q28) }\end{array}$ & 965 & 14,6 & 1173 & 18,1 & 1509 & 23,0 \\
\hline $\begin{array}{l}\text { Sistema respiratorio } \\
\text { (Q30-Q34) }\end{array}$ & 44 & 0,7 & 70 & 1,1 & 79 & 1,2 \\
\hline $\begin{array}{l}\text { Fisura de paladar } \\
\text { y labio leporino } \\
\text { (Q35-Q38) }\end{array}$ & 467 & 7,1 & 523 & 8,1 & 550 & 8,4 \\
\hline $\begin{array}{l}\text { Sistema digestivo } \\
\text { (Q39-Q45) }\end{array}$ & 322 & 4,9 & 324 & 5,0 & 340 & 5,2 \\
\hline $\begin{array}{l}\text { Órganos genitales } \\
\text { (Q50-Q56) }\end{array}$ & 157 & 2,4 & 201 & 3,1 & 189 & 2,9 \\
\hline $\begin{array}{l}\text { Sistema urinario } \\
\text { (Q60-Q64) }\end{array}$ & 297 & 4,5 & 396 & 6,1 & 487 & 7,4 \\
\hline $\begin{array}{l}\text { Sistema } \\
\text { osteomuscular } \\
(\mathrm{Q} 65-\mathrm{Q} 79)\end{array}$ & 1391 & 21,1 & 1693 & 26,2 & 1824 & 27,8 \\
\hline $\begin{array}{l}\text { Otras } \\
\text { malformaciones } \\
\text { congénitas } \\
\text { (Q80-Q89) }\end{array}$ & 26 & 0,4 & 61 & 0,9 & 86 & 1,3 \\
\hline $\begin{array}{l}\text { Anomalías } \\
\text { cromosómicas no } \\
\text { clasificadas en otra } \\
\text { parte (Q90-Q99) }\end{array}$ & 469 & 7,1 & 539 & 8,3 & 585 & 8,9 \\
\hline
\end{tabular}

Fuente: Sivigila, INS. 2015-2017. Estadisticas vitales, DANE. 2015 - 2017.

Tabla 4. Condición final y tasa de mortalidad por malformaciones congénitas. Colombia, 2015 - 2017.

\begin{tabular}{lccc}
\hline \multicolumn{1}{c}{ Condición final } & $\mathbf{2 0 1 5}$ & $\mathbf{2 0 1 6}$ & $\mathbf{2 0 1 7}$ \\
\hline Vivo & 4123 & 5449 & 5875 \\
Muerto & 871 & 1058 & 1049 \\
Tasa de mortalidad & $17,4 \%$ & $16,1 \%$ & $15,0 \%$ \\
\hline
\end{tabular}

Fuente: Sivigila, INS. 2015-2017. 


\section{Discusión}

El comportamiento de la notificación y la tasa de prevalencia para defectos congénitos reporta un incremento entre 2015 y 2017, lo que puede estar explicado por las alertas generadas desde el 2015 ante la epidemia del virus Zika y la posibilidad de desarrollar defectos congénitos de sistema nervioso central, que implicó el desarrollo de acciones de información, comunicación, educación y orientación técnica a todos los actores del sistema de salud y a la comunidad. Este escenario generó un aumento de la vigilancia de los defectos congénitos en el país para este periodo de tiempo ${ }^{9,17}$.

Con relación a las variables sociales y demográficas se observa que los defectos congénitos tienen mayor prevalencia en los menores de sexo masculino, lo que coincide con lo reportado en otros estudios ${ }^{18,19}$. Son hijos de madres mayores de 35 años, lo que ha sido afirmado por la literatura, donde se ha descrito que la edad materna avanzada es un factor de riesgo ${ }^{20,21}$ debido a tienen "mayor prevalencia de enfermedades crónicas, mayor riesgo de no disyunción cromosómica y de modificaciones genéticas en las células germinales, así como mayor probabilidad de cambios del medio uterino" 22 .

La mayor tasa de prevalencia de defectos congénitos se ubica en los casos sin afiliación al sistema de seguridad social en salud con pertenencia étnica indígena, lo que hace visible las bajas condiciones sociales y económicas en que vive y se desarrolla la mujer gestante y su familia. Estas variables son consideradas condiciones de vulnerabilidad que limitan posiblemente la falta de oportunidad y acceso a los servicios de salud ${ }^{19}$, lo que impacta en la supervivencia, discapacidad y/o mortalidad, siendo determinantes sociales que reflejan la distribución desigual del poder y los recursos en los sistemas socioeconómicos, políticos y culturales, lo que se traduce en consecuencias para la salud, mayor carga de la morbimortalidad y exposición diferencial a los riesgos para la salud ${ }^{23,24}$.

De acuerdo con el estudio de Pachajoa, et al. ${ }^{19}$ las estimaciones de las prevalencias de defectos congénitos que se han calculado en Colombia, oscilan entre 1,7 y 7,4 por 100 nacidos vivos, cifras identificadas en estudios realizados en hospitales de alta complejidad de ciudades como Cali, Barranquilla, Cartagena y Bogotá, no obstante, estas cifras pueden presentar un subregistro significativo teniendo en cuenta que no contemplan la integración de la información de la vigilancia en instituciones de diferentes niveles de atención, por lo que la prevalencia del evento no se podría extrapolar a un territorio.

Por otro lado, según el estudio de García Acero y Plata $^{25}$ sobre las anomalías congénitas en Colombia 2010-2013 las entidades territoriales que presentan una mayor tasa de prevalencia por 10.000 nacidos vivos fueron Cauca $(81,98)$, Arauca $(75,58)$, Tolima $(68,56)$, Huila $(64,82)$ y Putumayo $(63,26)$, datos que difieren del comportamiento presentado en 2015 a 2017. Teniendo en cuenta lo anterior y que la notificación de las malformaciones congénitas en Colombia inició en el año 2010 en 27 departamentos, en el año 2011 aumento a 30 departamentos y a partir del año 2012 la notificación se realiza en todo el país, podría inferirse que existe un subregistro inicial relacionado con la puesta en marcha del sistema de vigilancia para los defectos congénitos a nivel nacional.

De acuerdo al estudio de Nazer y Cifuentes ${ }^{26}$ en lo reportado por ECLAMC en el periodo 1995 - 2008 las tasas de prevalencia de malformaciones congénitas por 10.000 nacidos vivos más frecuentes para Colombia fueron la polidactilia $(36,3)$, seguida del pie equinovaro $(24,1)$, en cuarto lugar, las cardiopatías $(16,7)$ y en quinto lugar la hidrocefalia $(14,8)$. Al realizar la comparación con las tasas del ECLAMC se observa que las tasas de cardiopatías y de microcefalia son tasas inferiores al promedio latinoamericano, contrario a lo que pasa con las tasas de hidrocefalia, pie equinovaro y polidactilia las cuales se encuentran por encima del promedio.

Teniendo como referencia el estudio de Fernández y Zarante $^{27}$ desde el año 2001 para el ECLAMC, el cual se realiza en Bogotá, Cali, Ubaté, Manizales, Chiquinquirá y La Mesa, por grupo de malformación, el más frecuente fue el grupo de alteraciones osteomusculares con el 28,26\%, cifra muy cercana a la registrada por el presente estudio. El grupo de cardiopatías se ubicó en el séptimo lugar con el 6,14\% y el grupo de alteraciones del sistema nervioso se ubicó en el sexto lugar con el $8,48 \%$, en estos dos grupos las cifras difieren de las encontradas en el presente estudio lo cual puede deberse a lo mencionado anteriormente, la presencia de un subregistro significativo teniendo en cuenta que el ECLAMC es un estudio focalizado en unas instituciones de salud específicas y falta una integración a nivel nacional de otros datos que puedan aterrizar un poco más el comportamiento de las malformaciones congénitas. 
Al verificar más en detalle la frecuencia de defecto congénito en cada grupo, en el estudio de Fernández y Zarante $^{27}$ se encontró que el pie equinovaro y la polidactilia fueron las malformaciones más frecuentes y que coinciden con lo reportado en el presente estudio. En el caso de las cardiopatías congénitas no se especifica qué tipo de defecto se presentó, y en el grupo de los defectos de sistema nervioso se relaciona la hidrocefalia en el lugar quince de la clasificación de los más frecuentes.

En el estudio de Pachajoa, et al. ${ }^{19}$ los defectos congénitos más frecuentes son los cardiacos con el $20 \%$, seguido de los osteomusculares con el $17 \%$ y en cuarto lugar los defectos de sistema nervioso central con el 13\%, siendo este comportamiento similar a lo reportado en el presente estudio.

El aumento en el número de casos reportado en los años en estudio revela el impacto de la implementación de la vigilancia de defectos congénitos en el país, pese a esto, aún se requiere fortalecer los procesos de identificación de casos que garanticen la calidad de los registros y la correcta notificación de los mismos. En este sentido, la disponibilidad de registros poblacionales sobre este evento de interés en salud pública permite la identificación de un panorama que encamine la toma de acciones y la generación de políticas públicas que impacten tanto en la prevención de los defectos congénitos fortaleciendo las estrategias de consultas preconcepcionales y la mejora en la calidad de los controles prenatales, como una pronta identificación, diagnóstico, tratamiento y rehabilitación de los menores con algún tipo de defecto congénito.

El aumento en el comportamiento de la notificación y la tasa de prevalencia de los defectos congénitos para Colombia en los periodos analizados puede tener una relación con la epidemia del virus zika y su asociación con los defectos congénitos de sistema nervioso central, que generó el desarrollo de acciones intensificadas de vigilancia en salud pública para su prevención y control ${ }^{28}$.

Las características sociales y demográficas de los casos de defectos congénitos para Colombia son: sexo masculino, hijos de madres mayores de 34 años, etnia indígena y sin afiliación al sistema de seguridad social en salud. Lo anterior implica que los servicios de salud y los aseguradores realicen una gestión integral del riesgo desde la preconcepción para realizar búsqueda y valoración de riesgo de las mujeres en edad fértil orientados hacia la gestión de la afiliación, demanda inducida con promoción de la salud y detección temprana del riesgo ${ }^{29}$.

El tipo y la frecuencia de los defectos congénitos que se presentaron durante el periodo analizado, coincide, en términos generales con lo que ha sido reportado en el país por otros autores ${ }^{19,27}$.

Es importante continuar con los ejercicios de análisis del comportamiento de las malformaciones congénitas en el país de forma periódica, con el fin de consolidar una información más robusta que genere discusión, análisis y adicionalmente oriente políticas en salud pública que favorezcan el acceso a los servicios de salud y la calidad de vida de los menores con malformaciones congénitas.

Es necesario profundizar en el estudio de las malformaciones congénitas con el fin de identificar los factores de riesgo de los defectos que se identificaron como más frecuentes para los diferentes territorios del país, con el fin de realizar intervenciones en salud pública encaminadas a prevenir o mitigar la presencia del evento.

El grupo de especialistas involucrados en la detección temprana de los defectos congénitos tienen una enorme responsabilidad por ser quienes realizan el manejo inicial y en algunos casos la confirmación del diagnóstico, por lo que es necesario el fortalecimiento de las capacidades técnicas de los profesionales en el adecuado diagnóstico, evaluación de signos clínicos específicos, síndromes genéticos, etc. lo anterior debido a que el diagnóstico oportuno de un menor con defectos congénitos permitirá orientar su tratamiento de forma temprana el cual puede modificar de forma dramática su pronóstico.

\section{Consideraciones éticas}

El presente documento correspondió a un estudio sin riesgo de acuerdo con la Resolución 08430 de 1993, por ser descriptivo retrospectivo basado en la revisión de bases de datos; se respetó la confidencialidad de los datos individuales y la transparencia en la información, dado que no se realizó ninguna modificación intencionada de las variables.

\section{Conflicto de interés}

Las autoras declaran no tener conflictos de interés. 


\section{Referencias}

1. World Health Organization. WHO. 63 Asamblea Mundial De La Salud. Punto 11.7 del orden del día DEFECTOS CONGENITOS; 2010.

2. Alum JM, Fernández E, Moreno M, Ledesma E. Defectos congénitos mayores en nacidos vivos. Rev Arch Med Camagüey. 2015; 19 (2): 99-107.

3. Ortíz-Quiroga D, Ariza Y, Pachajoa H. Calidad de vida de familias de niños y adolescentes con discapacidad asociada a defectos congénitos. Rev Univ Psychol. 2018; 17(1): 161-170. doi: http://dx.doi.org/10.11144/javeriana.upsy17-1.cvfn.

4. European Surveillance of Congenital Anomalies (EUROCAT). Final Activity Report 2002-2003. University of Ulster Newtownabbey: Reino Unido. 2003; p. 12.

5. Matovelle C, Martínez Reyes F, Córdova Neyra F. Estudio Descriptivo: Frecuencia de Malformaciones Congénitas en Pacientes Pediátricos del Hospital "José Carrasco Arteaga". Rev Med HJCA 2015; 7(3): 249-253. doi: 10.14410/2015.7.3.ao.46.

6. Nazer HJ, Cifuentes OL. Malformaciones congénitas en Chile y Latino América: Una visión epidemiológica del ECLAMC del período 19952008. Rev Méd Chile. 2011; 139 (1): 72-78. doi: http://dx.doi.org/10.4067/S0034-98872011000100010.

7. Hernández Triguero $\mathrm{Y}$, Suárez Crespo M. Comportamiento de los defectos congénitos. Rev Ciencias Médicas. 2015; 19(1): 24-32.

8. García H. Malformaciones congénitas mayores: la necesidad del manejo multidisciplinario. Medigraphic. 2018; 85(3): 81-82.

9. Ministerio de Salud y Protección Social-Instituto Nacional de Salud. Circular conjunta 061 de 2015.

10. Ministerio de Salud y Protección Social-Instituto Nacional de Salud. Circular conjunta 043 de 2015.

11. Instituto Nacional de Salud. Circular externa 0004 de 2016.

12. Instituto Nacional de Salud. Circular externa 0007 de 2016.

13. Instituto Nacional de Salud. Circular externa 0017 de 2016.

14. Instituto Nacional de Salud. Circular externa 0020 de 2016.

15. Instituto Nacional de Salud. Circular externa 0022 de 2016.

16. Instituto Nacional de Salud. Circular externa 0063 de 2016.

17. Instituto Nacional de Salud. Circular externa 0064 de 2016

18. Lisi A, Botto LD, Rittler M, Castilla E, Bianca S, Bianchi F, et al . Sex and congenital malformations: An international perspective. Am J Med Genet A. 2005; 134A: 49-57. doi: http://dx.doi.org/10.1002/ajmg.a.30514.

19. Pachajoa H, Villota A, Cruz L, Ariza Y. Prevalencia de defectos congénitos diagnosticados en el momento del nacimiento en dos hospitales de diferente nivel de complejidad, Cali, Colombia, 2012-2013. Biomédica. 2015; 35( 2 ): 227-234. doi: http://dx.doi.org/10.7705/biomedica.v35i2.2295.

20. Nazer J, Cifuentes L. Prevalencia de malformaciones congénitas en hijos de madres mayores de 34 años y adolescentes. Hospital Clínico de la Universidad de Chile, 2002-2011. Rev Chil Obstet Ginecol. 2013; 78(4): 298-303.

21. Martinez-Galeano JM. La maternidad en madres de 40 años. Rev Cub Salud Pública. 2016; 42(3): 451-458.

22. Bravo-Gallego L, Teherán-Bravo J, Pantoja-Chamorro F, Díaz-Castro R, Acosta-Aragón M. Factores asociados a anomalías congénitas en neonatos del Cauca. Rev Pediatría. 2012; 45(1): 47-58. doi: https://doi.org/10.1016/S0120-4912(15)30005-7.

23. Organización Panamericana de la Salud. Determinantes e inequidades en salud. Rev Salud en las Américas. 2012; 2: 12-59.

24. Bernal DP, Cardona D. Caracterización de la mortalidad perinatal en Manizales, Colombia, 20092012. Hacia Promoc Salud. 2014; 19(2): 66-80.

25. García Acero M, Plata S. Caracterización de las anomalías congénitas desde la inclusión en el sistema de vigilancia Sivigila en Colombia, 2010-2013. Rev Univ Med. 2017; 58(1). doi: https://doi.org/10.11144/Javeriana.umed58-1.caci.

26. Nazer HJ, Cifuentes OL. Malformaciones congénitas en Chile y Latinoamerica: Una visión epidemiológica del ECLAMC 1995-2008. Rev Med Chil. 2011; 139: 72-78. doi: http://dx.doi.org/10.4067/S0034-98872011000100010.

27. Fernández N, Zarante I. Prevalencia y escala pronóstico para malformaciones congénitas en Colombia: La responsabilidad de pediatras y neonatólogos. Registro de 54.397 nacimientos. Rev UCIN. 2008; 1: 28-32.

28. Alvarado Socarras JL. Zika en neonatos: Todo no está dicho. Rev Univ Ind Santander Salur 2016;48(2):160-162.

29. Martinez-Galeano JM. La maternidad en madres de 40 años. Rev Cub Salud Pública. 2016; 42 (3): 451-458. 